\title{
DNA Origami Model for Simple Image Decoding
}

\author{
Risheng Wang, ${ }^{1}$ Zhixiang Yin $\mathbb{D}^{2},{ }^{2}$ Jianzhong Cui, ${ }^{1}$ Jing Yang, ${ }^{1}$ Zhen Tang, ${ }^{1}$ Xinmu Yang, \\ and Xianya Geng ${ }^{1}$
}

${ }^{1}$ Anhui University of Science and Technology, Huainan, China

${ }^{2}$ Shanghai University of Engineering Science, Songjiang, China

Correspondence should be addressed to Zhixiang Yin; zxyin66@163.com

Received 24 April 2020; Accepted 18 May 2020; Published 3 August 2020

Academic Editor: Jia-Bao Liu

Copyright ( 2020 Risheng Wang et al. This is an open access article distributed under the Creative Commons Attribution License, which permits unrestricted use, distribution, and reproduction in any medium, provided the original work is properly cited.

DNA origami is the application of self-assembly in nanotechnology. The combination of DNA origami and hybridization chain reaction is one of the important application methods of DNA origami. In this paper, DNA origami is used to design the cipher pattern on the base of origami. The cipher chain, which is put into the reaction solution, hybridizes with the molecular beacon and the hairpin structure that form the cipher pattern to build a DNA origami model that can decode the pattern. The cipher chain consists of the starting chain and the intermediate chain. When the cipher is correct, the cipher chain can open the molecular beacon and the hairpin structure to display the cipher pattern on the origami base in the solution.

\section{Introduction}

DNA origami is a DNA self-assembly method proposed in recent years. The concept of DNA origami was proposed firstly by Rothemund [1]. Compared with traditional DNA self-assembly, DNA origami is easier in constructing highly complex and structurally stable nanostructures. It also has addressability, which is a significant progress in the field of DNA self-assembly and DNA nanotechnology. Through DNA origami technology, Qian and his team successfully constructed the map of China, further proving that DNA origami has the ability to construct almost any complex twodimensional nanoshape [2]. In 2011, Han et al. studied the stereoscopic vase structure that can precisely regulate the three-dimensional surface and pushed the research of DNA origami in the structural design and manufacturing to the climax $[3,4]$. In 2013, the bipedal DNA Walker played a key role in the research of DNA origami "orbit" [5]. In 2014, DNA origami robots were designed for conventional computing [6]. In the same year, Koirala and his team combined DNA origami nanostructures to mechanochemical sensors [7]. In 2017, Tikhomirov et al. used the square DNA origami as the basic unit to obtain the pattern of the Mona Lisa at the micron level, further proving the ability of DNA origami to construct any complex pattern, indicating the addressability of DNA origami [8]. In 2018, Chao combined DNA origami with hybridization chain reaction to solve the maze problem [9]. The substrate constructed by DNA origami has great advantages in assembling functional carbon nanotubes, nanoparticles, and proteins. Many complex and diverse nanostructures have been designed and assembled [10-12]. DNA origami can construct highly complex nanostructures, which has great potential in the "nano" field. Wang and his team used DNA origami nanostructures to visualize cell uptake and metastasis in tumor cells [13]. In fact, DNA origami has been applied to single molecule detection [14, 15] and logic operation $[16,17]$. DNA origami has been used as the carbon nanotubes, protein structure, and the basic template for the assembly of functional metal nanoparticles [18-22].

The combination of DNA origami and hybridization chain reaction is one of the important application methods of DNA origami. Hybridization chain reaction (HCR) is a new signal amplification method based on DNA hybridization proposed by Dirks and Pierce in 2004. This is an isothermal amplification technology, which greatly simplifies the requirements of the instrument. It only needs simple temperature control equipment such as heating 
module and water bath pot to complete the signal amplification of hybridization chain reaction [23]. When there are only two kinds of DNA strands in the test tube solution, they are stable in the solution and will not be hybridized and opened. Once the starting DNA strand is added, the two kinds of DNA strands will be hybridized alternately to form a long double stranded DNA polymer with a gap. The use of hybridization chain reaction can not only amplify the signal of single strand DNA, but also amplify the signal of other biological molecules. It can induce oligonucleotides to hybridize with each other using a small segment of nucleotide chain as initiator to form a single strand DNA with twodimensional or three-dimensional spatial structure by designing different oligonucleotides reasonably [23, 24]. At present, hybridization chain reaction has been widely used in nucleic acid, protein detection, biosensor, and other fields [25-29]. Dong and his team combined the hybridization chain reaction with G-quadruplex to construct a fluorescent sensor for target DNA unmarked detection. The target DNA can be detected by changing the fluorescence intensity before and after the reaction [30]. Xiao et al. designed a variety of chemiluminescence imaging technologies by combining DNA microarray with hybridization chain reaction amplification induced by adjacent binding and used them in sensitive screening and detection of protein biomarkers [31]. Li et al. proposed a method to light up the fluorescent sensor by using hybridization chain reaction and DNA three-strand assembly [32]. Because of the advantages of simple operation and low cost, this method has high application value in biomarker determination, clinical diagnosis, and biomedical detection.

We use DNA origami to design the cipher pattern on the base of origami. We put the cipher chain into the reaction solution, which carry on the hybridization chain reaction with the molecular beacon and the hairpin structure that form the cipher pattern. It could establish the DNA origami model, which can decode the pattern. It has been proved that hybridization chain reaction and DNA origami structure have the good application prospect in this field [33-46]. The cipher chain is composed of the starting chain and the intermediate chain containing the password. Through the reaction with the origami base containing the cipher pattern in the solution, the pattern is displayed when the cipher is correct. The pattern is observed by imaging with atomic force microscope.

\section{DNA Origami and Hybridization Chain Reaction}

2.1. DNA Origami. DNA origami refers to folding a long M13mp18 phage DNA strand back and forth as a scaffold chain and using multiple staples to fix the shape to obtain fine and complex two-dimensional structure. Compared with the self-assembly of DNA module, the design of DNA strand in DNA origami is relatively simple. It also have high assembly efficiency, nano-addressable characteristics (realized by redesigning the single strand at the designated position of staple chain and scaffold chain), and the ability to construct more complex and fine patterns. The substrate constructed by DNA origami has great advantages in assembling functional carbon nanotubes, nanoparticles, proteins, and so on.

\subsection{Hybridization Chain Reaction. Hybridization chain} reaction (HCR) is a new signal amplification method proposed by Dirks and Pierce. It can induce oligonucleotides to hybridize with each other to form DNA with spatial structure using a small segment of nucleotide chain as initiator by rationally designing different oligonucleotides. The hybridization chain reaction is to induce DNA molecules of two different types of hairpin structures to hybridize alternately by using the starting chain, open the hairpin structure alternately, and form a double strand DNA nanowire with a gap. And this process does not need enzyme participation; the reaction conditions are mild; the operation is simple. The reaction principle is shown in Figure 1.

$C_{1}$ and $C_{2}$ are two DNA strands of hairpin structure, which can exist in solution at the same time and can remain stable without any hybridization reaction. $C_{1}$ and $C_{2}$ are all composed of three parts, the sticky end, the "stem" in the form of double chain, and the ring part of single chain, as shown in Figure $1(1)$. Among them, $b$ and $b^{*}$ are base complementary and exist in the form of double chain, called the stem of $C_{1}\left(C_{2}\right)$ chain; $a$ is the single chain sticky extending from the "stem" of $C_{1}$ chain, and the single chain segment $c$ of hairpin is called the ring of $C_{1}$ chain; accordingly, $a^{*}$ is the $C_{2}$ chain ring, and $c^{*}$ is the single chain sticky end extending from the "stem" of $C_{2}$ chain. We can call $T$ initiation chain, which is a DNA single strand composed of $a^{*}$ and $b^{*}$ parts. When the initiation chain $T$ is added, the $a^{*}$ and $b^{*}$ segments hybridize with the sticky end $a$ and stem $b$ of $C_{1}$, respectively. The hairpin segment of $C_{1}$ is opened, and the segment $c-b^{*}$ is exposed in the form of a single chain, as shown in Figure 1 (2). The segment $c-b^{*}$ exposed by $C_{1}$ and segment $c^{*}-b$ of $C_{2}$ have a hybridization chain reaction. The hairpin section of $C_{2}$ is opened, and the segment $a^{*}-b^{*}$ is exposed by $C_{2}$, as shown in Figure 1 (3). The exposed segment $a^{*}-b^{*}$ of $C_{2}$ continues to hybridize with the next $C_{1}$ and open the hairpin structure. It will circulate and react successively until the $C_{1}$ and $C_{2}$ in the solution are exhausted. Finally, alternating hybridization of $C_{1}$ and $C_{2}$ assembled spontaneously a long DNA nanowire. Each initiation chain $T$ is equivalent to the growth site of a DNA nanowire. With the concentration of "fuel" $C_{1}$ and $C_{2}$ being constant and sufficient, the higher the concentration of the initiation chain $T$ is, the shorter the nanowire is and the smaller the molecular weight is.

\section{DNA Origami Model}

3.1. Composition of the Model. Because of the simple image design, we give a simple cipher image circuit diagram of "Tian," shaped as the Chinese character "Field."

There are three lines in the horizontal and vertical direction of the circuit diagram, and each line has two short paths (two short edges) with a total of 12 edges. The 12 edges of the circuit diagram can be used to design simple cipher 


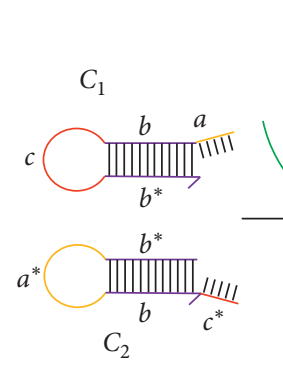

(1)

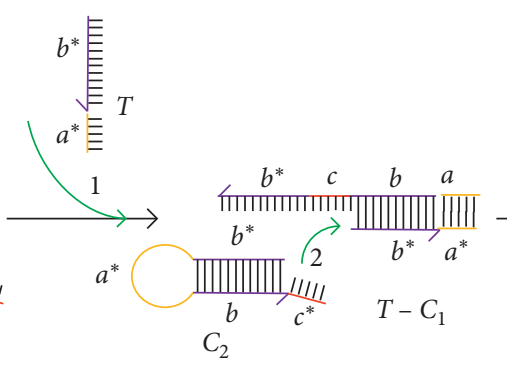

(2)
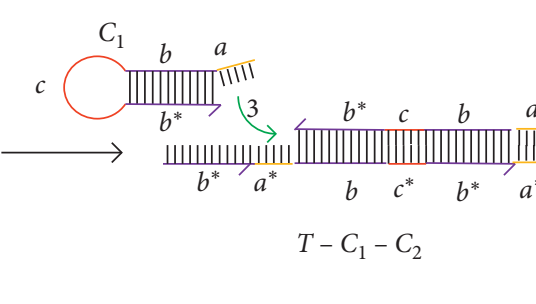

(3)

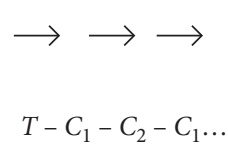

(4)

FIGURE 1: Basic principle of hybridization chain reaction.

image. The circuit diagram is mapped on the origami base, which is composed of several molecular beacons and ordinary hairpin structure fixed on the DNA origami base. Among them, there are nine ordinary hairpin structures at the vertices and intersections of the circuit diagram, which are fixed on the origami base in the form of three in each row and three rows in total; there are molecular beacons with a fluorescence group and a quenching group on each edge (between the two adjacent hairpin structures) of the circuit diagram, a total of 12, as shown in Figure 2(a). We use the short chain to fold the circular phage into a two-dimensional rectangle as the origami base (shown in gray in the figure). At a certain distance on the origami base, we extend the staple chain with the sticky end (shown in light blue in the figure), which is connected with the hairpin structures (including molecular beacon and ordinary hairpin structure). The red one is the molecular beacon with fluorescence and quenching group, and the dark blue one is the ordinary hairpin structure.

The structure of molecular beacon and hairpin structure is basically the same. They are all composed of five-segment oligonucleotide fragments, with two segments that complement each other to form the "stems" of the chain. One is the "ring" of the chain, and the other is complementary to the sticky end of a stapling chain extending on the origami base, which fixes the chain on the origami base. Only compared with the ordinary hairpin structure, the two sticky ends of the molecular beacon have luminescent fluorescence groups and quenching groups. They do not light up when they are not turned on. And they only turn on and shine when the starting chain and the intermediate auxiliary chain (collectively referred to here as "the cipher chain") are added. And the ordinary hairpin structure plays a role of route guidance to avoid causing other neighboring molecular beacons to open in advance when opening these molecular beacons, so that it can be opened according to the designed route. In this way, the molecular beacons fixed on the origami base can be used for simple cipher image design, and the corresponding molecular beacons need to be opened to display the cipher pattern.

To open the corresponding molecular beacon, a cipher chain needs to be added, which is composed of a starting chain and an intermediate chain, as shown in Figure 2(b). The starting chain consists of two oligonucleotide fragments, as shown in the left side of Figure 2(b); the intermediate chain consists of four oligonucleotide fragments, as shown in the right side of Figure 2(b). Only the chain containing the correct fragment can open the corresponding molecular beacon, and then the cipher pattern can be displayed.

3.2. The Construction of Cipher and the Realization of Decoding. According to the composition of the model, we designed a cipher pattern and according to the cipher pattern, we designed the base sequence of hairpin structure (including molecular beacon and hairpin structure) on origami base. The base sequence of each hairpin structure passed by the pattern is almost the same. It is only different from the base sequence of molecular beacon and hairpin structure at the binding marker, and the base sequence of hairpin structure of the starting point at the junction of the starting chain, as shown in Figure 3(a). We set the top left as the starting point of the cipher pattern, and the cipher pattern we designed is the dotted brown line on the graph below. The base sequence of hairpin structure in other positions is different from it, so it will not react with it and open it.

In the cipher pattern on this origami substrate, all hairpin structures can be regarded as consisting of five segments of oligonucleotide fragments. One of the segments is complementary to the sticky end of a staple chain extending on the origami base and is not marked with letters. The remaining four segments are marked with letters as shown in the hairpin structure on Figure 3(a). Segments $a$ and $a^{*}$ are the stems of the hairpin structure, and they are complementary; segment $c$ is the loop of the hairpin structure. The above oligonucleotide fragments are the same in all the hairpin structures. Only compared to the ordinary hairpin structure, the two sticky ends of the $a$ and $a^{*}$ segments of the molecular beacon have luminescent fluorescent groups and quenching groups. The hairpin structure of the starting point is different from other hairpin structures in the oligonucleotide fragment combined with the starting chain, and we mark it as the $x$ segment. We mark other hairpin structures of this segment as the $e$ segment.

The base sequence of the hairpin structure in the position of noncipher pattern is different from that in the position of cipher pattern, so that the cipher chain will not react with it and this hairpin structure will not be opened. In order to simplify the design of hairpin structure, we can only design different noncipher pattern hairpin structure adjacent to the 


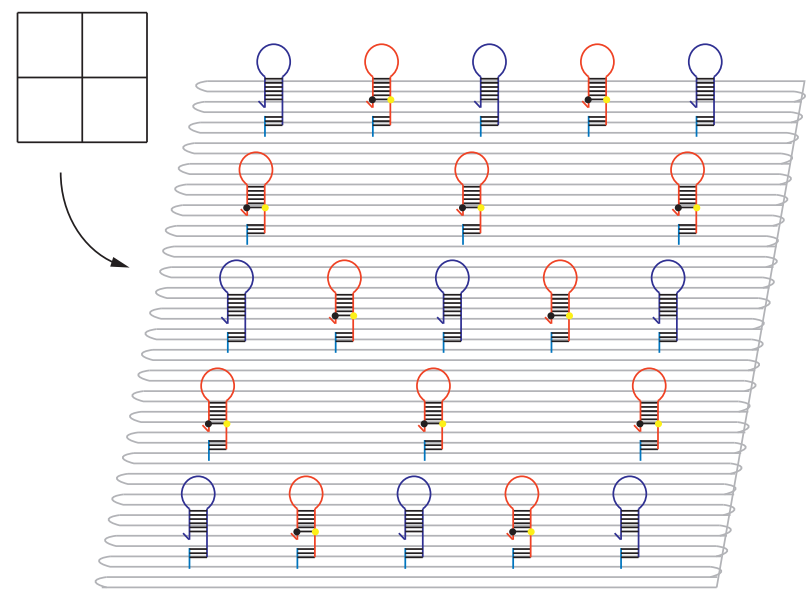

(a)

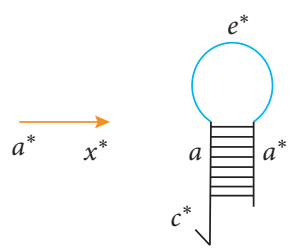

(b)

FIgURE 2: (a) Circuit diagram of origami base and hairpin structure; (b) cipher chain, consisting of starting chain (left) and intermediate chain (right).

hairpin structure at the location of cipher pattern to avoid other hairpin structures participating in the reaction.

We put the designed origami base into the solution in advance. Then we add the cipher chain to the solution. The hairpin structure of the starting point has a special oligonucleotide fragment, which can be specifically combined with the starting chain in the cipher chain, thereby ensuring the uniqueness of the starting point.

When the cipher chain is added to the solution, the starting chain will open the hairpin structure of the starting point. The opening process is shown in Figure 3(b). The $x^{*}$ segment of the starting chain will complement and pair with the $x$ segment of the starting point hairpin structure, and the $a^{*}$ segment of the starting chain will complement and pair with the $a$ segment of the starting point hairpin structure, thereby opening the hairpin structure. The hairpin $c$ segment and $a^{*}$ segment of the hairpin structure will be complementarily paired with the intermediate chain $c^{*}$ segment and the a segment of the cipher chain, thereby opening the intermediate chain. The loop $e^{*}$ segment and $a^{*}$ segment of the intermediate chain can continue to be complementary paired with the $e$ segment and $a$ segment of the molecular beacon, thereby opening the molecular beacon to make the pattern glow.

Another molecular beacon adjacent to the starting point hairpin structure will not open (that is, the cipher pattern will not pass through) because its base sequence structure is different from the intermediate chain. In this way, according to the designed cipher pattern, the hairpin structure can be opened in sequence; the starting chain can open the hairpin structure of the starting point; the hairpin structure opens the intermediate chain; the intermediate chain is opening the molecular beacon; the molecular beacon lights up; and then the molecular beacon opens the intermediate chain so as to finally display the cipher pattern, as shown in Figure 3(c). At the same time, the intermediate chain can only open the adjacent hairpin structure of the previous one, thereby avoiding mismatches.

The circuit diagram of cipher pattern we designed does not directly arrange the molecular beacons in order. The addition of ordinary hairpin structures between the molecular beacons can avoid the situation that there is a fold back in the path of cipher pattern and the opening sequence cannot follow the design image. As shown in Figure 4(a), $T_{1}$ goes through $T_{2}$ to $T_{3}$ instead of directly from $T_{1}$ to $T_{3}$. The correct and wrong opening methods are shown in Figure 4(b).

We pour the cipher chain into the solution. If the cipher chain is correct, the starting chain can open the hairpin structure at the starting point, and then the intermediate chain of the cipher chain can sequentially open the hairpin structure that the cipher pattern passes through. And the opened molecular beacon will light up to display the cipher pattern. If the cipher is not correct, no matter the base sequence in the starting chain or the intermediate chain is wrong, the hairpin structure cannot be opened to display the cipher pattern. As shown in Figure 4(c), the upper part is the open figure with correct cipher chain display, and the lower part is the figure with wrong cipher chain display (this is the starting chain error).

We also consider hiding the base code of the intermediate chain, and the correct code is its password. Since the encryption algorithm of DNA coding has been very mature, we hide the base sequence of intermediate chain. After only obtaining its decoding method, completing the decoding, and finding the corresponding intermediate chain to match, we could get the correct cipher pattern while putting the cipher chain into the solution. If the decoding is not successful, we will not get the final correct cipher pattern. In this way, we can make the cipher chain more secure. We should have not only the cipher chain but also the correct method for decoding the cipher chain, so 

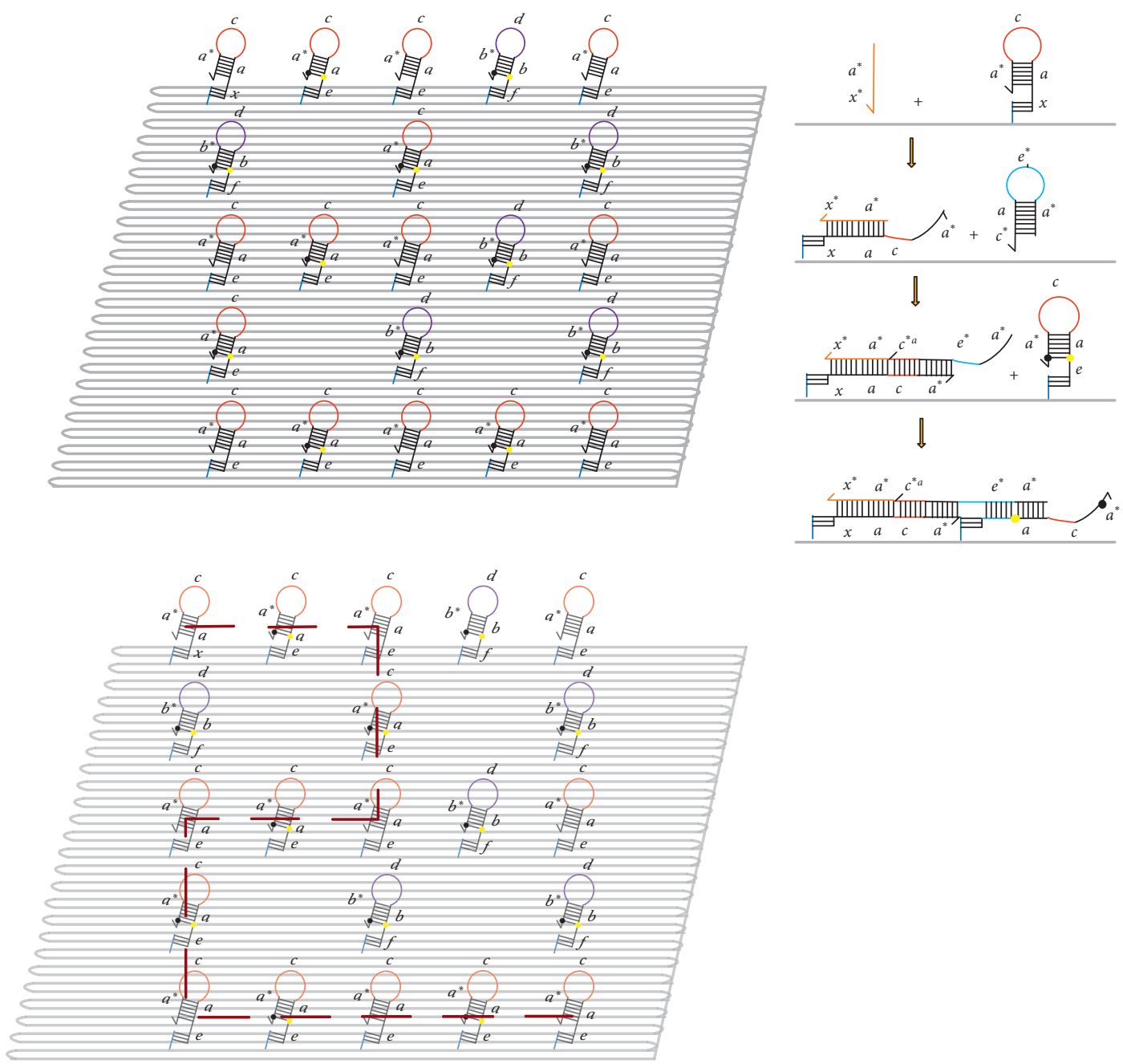

(a)

(b)

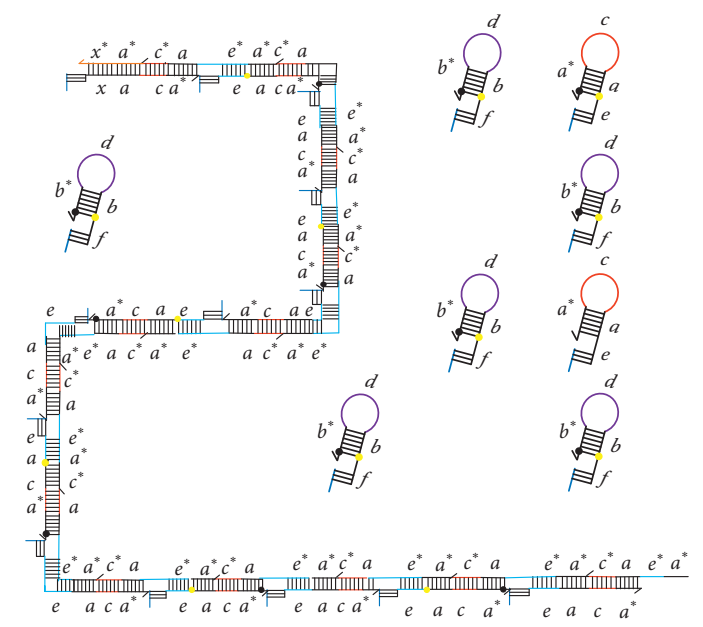

(c)

FIgURE 3: (a) The hairpin structure and cipher pattern designed on origami base; (b) the opening process after adding the cipher chain; (c) the cipher pattern after opening. 


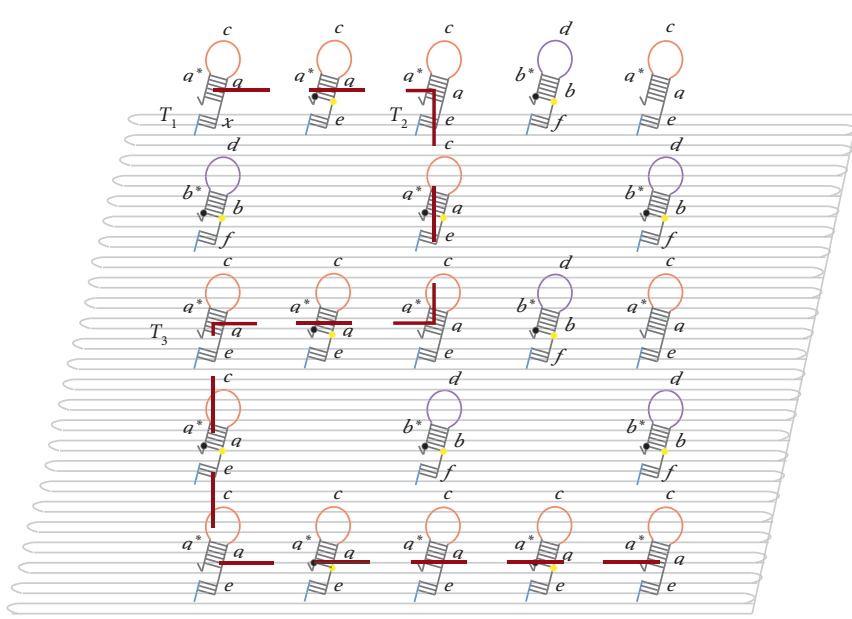

(a)

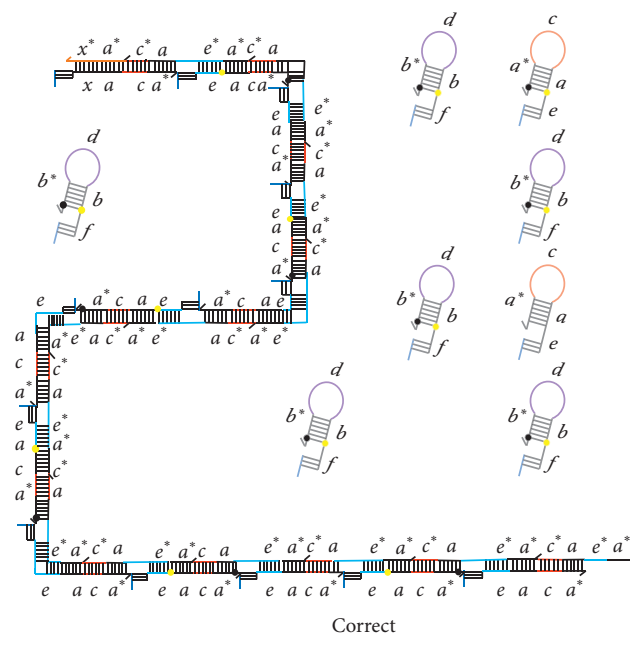

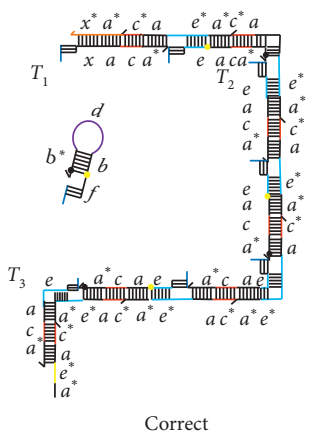

Correct

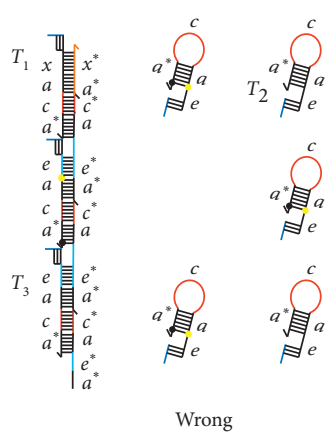

(b)

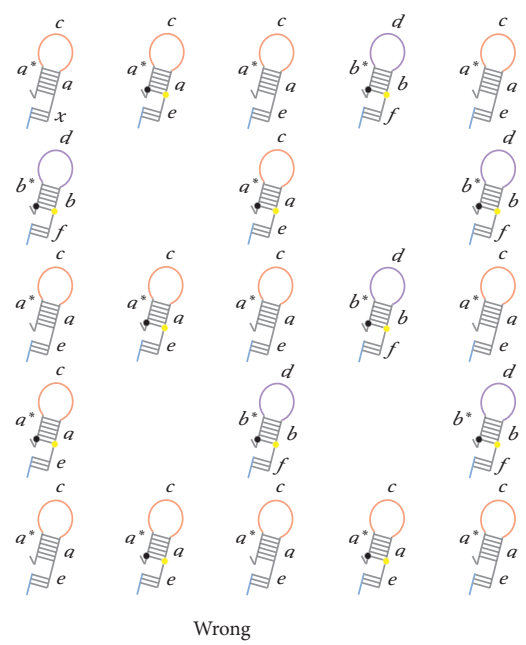

(c)

Figure 4: (a) Cipher pattern to be displayed on the origami base; (b) the correct and wrong ways to open the designed cipher diagram in the reaction; (c) origami base figure after adding cipher chain to react.

that the correct answer can form the correct path. This is what we will do next.

\section{Conclusion}

In this paper, DNA origami model for simple image decoding is established by combining DNA origami with hybridization chain reaction. We design the hairpin structure on the origami base, which can decode the simple cipher pattern. The correct cipher chain composed of a starting chain and an intermediate chain can open the molecular beacon and display the cipher pattern. But the hairpin structure is a one-time channel, which cannot be reused; the cipher pattern cannot be designed to crossovers. With the development of molecular biology and bioengineering, the method is expected to have more progress in reusability and can further be expanded to solve more complex decoding problems.

\section{Data Availability}

The data used to support the findings of this study are available from the corresponding author upon request.

\section{Conflicts of Interest}

The authors declare that they have no conflicts of interest.

\section{References}

[1] P. W. K. Rothemund, "Folding DNA to create nanoscale shapes and patterns," Nature, vol. 440, no. 7082, pp. 297-302, 2006.

[2] L. Qian, Y. Wang, Z. Zhang et al., "Analogic China map constructed by DNA," Chinese Science Bulletin, vol. 51, no. 24, pp. 2973-2976, 2006.

[3] D. Han, S. Pal, J. Nangreave, Z. Deng, Y. Liu, and H. Yan, "DNA origami with complex curvatures in three-dimensional space," Science, vol. 332, no. 6027, pp. 342-346, 2011. 
[4] D. Han, S. Pal, Y. Yang et al., "DNA gridiron nanastructures based on four-arm junctions," Science, vol. 339, no. 6126, pp. 1412-1415, 2011.

[5] T. E. Tomov, R. Tsukanov, M. Liber, R. Masoud, N. Plavner, and E. Nir, "Rational design of DNA motors: fuel optimization through single-molecule fluorescence," Journal of the American Chemical Society, vol. 135, no. 32, pp. 11935-11941, 2013.

[6] Y. Amir, E. Ben-Ishay, D. Levner, S. Ittah, A. Abu-Horowitz, and I. Bachelet, "Universal computing by DNA origami robots in a living animal," Nature Nanotechnology, vol. 9, no. 5, pp. 353-357, 2014.

[7] D. Koirala, P. Shrestha, T. Emura et al., "Single-molecule mechanochemical sensing using DNA origami nanostructures," Angewandte Chemie International Edition, vol. 53, no. 31, pp. 8137-8141, 2014.

[8] G. Tikhomirov, P. Petersen, and L. Qian, "Fractal assembly of micrometre-scale DNA origami arrays with arbitrary patterns," Nature, vol. 552, no. 7683, pp. 67-71, 2017.

[9] J. Chao, J. Wang, F. Wang et al., "Solving mazes with singlemolecule DNA navigators," Nature Materials, vol. 18, no. 3, pp. 273-279, 2018.

[10] F. Zhang, J. Nangreave, Y. Liu, and H. Yan, "Reconfigurable DNA origami to generate quasifractal patterns," Nano Letters, vol. 12, no. 6, pp. 3290-3295, 2012.

[11] B. Wei, M. Dai, and P. Yin, "Complex shapes self-assembled from single-stranded DNA tiles," Nature, vol. 485, no. 7400, pp. 623-626, 2012.

[12] Y. Ke, J. Sharma, M. Liu, K. Jahn, Y. Liu, and H. Yan, "Scaffolded DNA origami of a DNA tetrahedron molecular container," Nano Letters, vol. 9, no. 6, pp. 2445-2447, 2009.

[13] P. Wang, M. A. Rahman, Z. Zhao et al., "Visualization of the cellular uptake and trafficking of DNA origami nanostructures in cancer cells," Journal of the American Chemical Society, vol. 140, no. 7, 2018.

[14] M. Endo, Y. Katsuda, K. Hidaka, and H. Sugiyama, "A versatile DNA nanochip for direct analysis of DNA base-excision repair," Angewandte Chemie International Edition, vol. 49, no. 49, pp. 9412-9416, 2010.

[15] Y. Sannohe, M. Endo, Y. Katsuda, K. Hidaka, and H. Sugiyama, "Visualization of dynamic conformational switching of the G-quadruplex in a DNA nanostructure," Journal of the American Chemical Society, vol. 132, no. 46, pp. 16311-16313, 2010.

[16] C. Zhang, J. Yang, S. Jiang, Y. Liu, and H. Yan, "DNAzymebased logic gate-mediated DNA self-assembly," Nano Letters, vol. 16, no. 1, pp. 736-741, 2015.

[17] D. Wang, Y. Fu, J. Yan et al., "Molecular logic gates on DNA origami nanostructures for MicroRNA diagnostics," Analytical Chemistry, vol. 86, no. 4, pp. 1932-1936, 2014.

[18] J. Sharma, R. Chhabra, Y. Liu, Y. Ke, and H. Yan, "DNAtemplated self-assembly of two-dimensional and periodical gold nanoparticle arrays," Angewandte Chemie International Edition, vol. 45, no. 5, pp. 730-735, 2006.

[19] M. Pilo-Pais, S. Goldberg, E. Samano, T. H. Labean, and G. Finkelstein, "Connecting the nanodots: programmable nanofabrication of fused metal shapes on DNA templates," Nano Letters, vol. 11, no. 8, pp. 3489-3492, 2011.

[20] H. Yan, S. H. Park, and G. Finkelstein, "DNA-templated selfassembly of protein arrays and highly conductive nanowires," Science, vol. 301, no. 5641, pp. 1882-1884, 2003.

[21] N. V. Voigt, T. Tørring, A. Rotaru et al., "Single-molecule chemical reactions on DNA origami," Nature Nanotechnology, vol. 5, no. 3, pp. 200-203, 2010.
[22] H. T. Maune, S.-p. Han, R. D. Barish et al., "Self-assembly of carbon nanotubes into two-dimensional geometries using DNA origami templates," Nature Nanotechnology, vol. 5, no. 1, pp. 61-66, 2010.

[23] R. M. Dirks and N. A. Pierce, "From the Cover: triggered amplification by hybridization chain reaction," Proceedings of the National Academy of Sciences, vol. 101, no. 43, pp. 15275-15278, 2004.

[24] D. Evanko, "Hybridization chain reaction," Nature Methods, vol. 1, no. 3, p. 186, 2004.

[25] D. Peng and R. Yuan, "A novel electrochemical aptasensor for thrombin detection based on hybridization chain reaction as enhanced signals," Chemical Sensors, vol. 34, no. 4, pp. 14-19, 2014.

[26] B. Zhang, B. Liu, D. Tang, R. Niessner, G. Chen, and D. Knopp, "DNA-based hybridization chain reaction for amplified bioelectronic signal and ultrasensitive detection of proteins," Analytical Chemistry, vol. 84, no. 12, pp. 53925399, 2012.

[27] Y. Chen, J. Xu, J. Su, Y. Xiang, R. Yuan, and Y. Chai, "In situ hybridization chain reaction amplification for universal and highly sensitive electrochemiluminescent detection of DNA," Analytical Chemistry, vol. 84, no. 18, pp. 7750-7755, 2012.

[28] J. Huang, Y. Wu, Y. Chen et al., "Pyrene-excimer probes based on the hybridization chain reaction for the detection of nucleic acids in complex biological fluids," Angewandte Chemie International Edition, vol. 50, no. 2, pp. 401-404, 2011.

[29] D. Yang, Y. Tang, and P. Miao, "Hybridization chain reaction directed DNA superstructures assembly for biosensing applications," TrAC Trends in Analytical Chemistry, vol. 94, pp. 1-13, 2017.

[30] J. Dong, X. Cui, Y. Deng, and Z. Tang, “Amplified detection of nucleic acid by G-quadruplex based hybridization chain reaction," Biosensors and Bioelectronics, vol. 38, no. 1, pp. 258-263, 2012.

[31] Q. Xiao, P. J. Wu, and H. Ju, "Multiplexed chemiluminescence imaging assay of protein biomarkers using DNA microarray with proximity binding-induced hybridization chain reaction amplification," Analytica Chimica Acta, vol. 1032, no. 1032, pp. 130-137, 2018.

[32] Z. Li, L. Tingting, and S. Ruidi, "A label-free light-up fluorescent sensing platform based upon hybridization chain reaction amplification and DNA triplex assembly," Talanta, vol. 189, pp. 137-142, 2018.

[33] Z. Zhang, Y. Yang, F. Pincet, M. C. Llaguno, and C. Lin, "Placing and shaping liposomes with reconfigurable DNA nanocages," Nature Chemistry, vol. 9, no. 7, pp. 653-659, 2017.

[34] N. Y. Wong, H. Xing, L. H. Tan, and Y. Lu, "Nano-encrypted morse code: a versatile approach to programmable and reversible nanoscale Assembly and disassembly," Journal of the American Chemical Society, vol. 135, no. 8, pp. 2931-2934, 2013.

[35] S. D. Mason, Y. Tang, Y. Li, X. Xie, and F. Li, "Emerging bioanalytical applications of DNA walkers," TrAC Trends in Analytical Chemistry, vol. 107, pp. 212-221, 2018.

[36] Z. Li and K. Bošković, "Nanopore-based DNA hard drives for rewritable and secure data storage," Nano Letters, vol. 20, no. 5, pp. 3754-3760, 2020.

[37] T. G. W. Edwardson, K. L. Lau, D. Bousmail, C. J. Serpell, and H. F. Sleiman, "Transfer of molecular recognition information from DNA nanostructures to gold nanoparticles," Nature Chemistry, vol. 8, no. 2, pp. 162-170, 2016.

[38] S. Shah, A. K. Dubey, and J. Reif, "Improved optical multiplexing with temporal DNA barcodes," ACS Synthetic Biology, vol. 8, no. 5, pp. 1100-1111, 2019. 
[39] M. Xiao, W. Lai, T. Man et al., "Rationally engineered nucleic acid architectures for biosensing applications," Chemical Reviews, vol. 119, no. 22, pp. 11631-11717, 2019.

[40] K. Chen, X. Li, and J. Yang, "Precise control of gold nanoparticles on DNA origami for logic operation," Journal of Nanomaterials, vol. 2019, pp. 1-9, 2019.

[41] J. O'Brien and A. Murugan, "Temporal pattern recognition through analog molecular computation," ACS Synthetic Biology, vol. 8, no. 4, pp. 826-832, 2019.

[42] G. Chatterjee, N. Dalchau, R. A. Muscat, A. Phillips, and G. Seelig, "A spatially localized architecture for fast and modular DNA computing," Nature Nanotechnology, vol. 12, no. 9, pp. 920-927, 2017.

[43] K. Sergio, W. J. Paschoalino, and L. T. Kubota, "Supramolecular DNA origami nanostructures for use in bioanalytical applications," TrAC Trends in Analytical Chemistry, vol. 108, pp. 88-97, 2018.

[44] K. E. Bujold, A. Lacroix, and H. F. Sleiman, "DNA nanostructures at the interface with biology," Chem, vol. 4, no. 3, pp. 495-521, 2018.

[45] T. Fu, Y. Lyu, and H. Liu, "DNA-based dynamic reaction networks[J]," Trends in Biochemical Sciences, vol. 43, no. 7, pp. 457-560, 2018.

[46] X. Song and J. Reif, "Nucleic acid databases and molecularscale computing," ACS Nano, vol. 13, no. 6, pp. 6256-6268, 2019. 